\title{
Métodos de avaliação da apraxia de fala na infância: revisão sistemática
}

\section{Methods of assessing of childhood apraxia of speech: systematic}

\author{
review
}

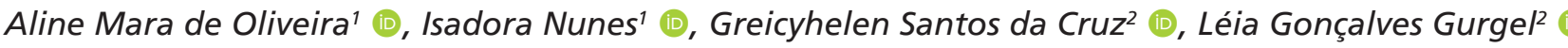

\section{RESUMO}

Objetivos: Revisar sistematicamente os protocolos e/ou avaliações que contribuem para o diagnóstico de apraxia de fala na infância (AFI) e classificá-los de acordo com a dimensão clínica avaliada. Estratégia de pesquisa: Estudo de revisão sistemática da literatura nas bases de dados MEDLINE (acessado via PubMed), LILACS, Scopus e SciELO, com os descritores Apraxias, Childhood apraxia of speech, Evaluation, Assessment, Validation Studies, Evaluation Studies, Language Therapy, Rehabilitation of Speech and Language Disorders, Child e Child, Preschool. Critérios de seleção: A busca nas bases de dados foi conduzida por três pesquisadores independentes. Foram incluídos estudos que avaliavam, de forma clara, sujeitos com suspeita ou diagnóstico de AFI. Os revisores realizaram a coleta de dados no que diz respeito às características metodológicas, intervenções e desfechos dos estudos, por meio de planilhas previamente elaboradas especificamente para o presente estudo. O dado principal coletado foi referente aos procedimentos de avaliação da AFI para crianças. Resultados: A maior parte dos estudos (14 dos 21 incluídos) realizou a associação entre a avaliação de habilidades motoras e/ou articulatórias e segmentais. Cinco realizaram avaliação de todos os aspectos elencados: motor e/ou articulatória, segmental e suprassegmental e dois realizaram apenas avaliação motora e/ou articulatória. A idade dos sujeitos variou de 3 a 12 anos. Conclusão: A maioria das pesquisas considerou a associação entre habilidades motoras e/ou articulatórias e segmentais para avaliação da apraxia de fala na infância. Sugere-se a realização de mais estudos, a fim de buscar evidências de validade.

Descritores: Reabilitação dos transtornos da fala e da linguagem; Fala; Inteligibilidade da fala; Apraxias; Criança

\begin{abstract}
Purpose: Systematically review the protocols and/or assessments that contribute to the diagnosis of CAS and classify them according to the clinical dimension evaluated Research strategy: Study of systematic literature review in the databases MEDLINE (accessed via PubMed), LILACS, Scopus and SciELO with the descriptors Apraxias, Childhood apraxia of speech, Evaluation, Assessment, Validation Studies, Evaluation Studies, Language Therapy, Rehabilitation of Speech and Language Disorders, Child and Child, Preschool. Selection criteria: The search for scientific articles in the databases was conducted by three independent researchers. Studies that clearly assessed subjects with suspected or diagnosed PIA were included. The reviewers performed data collection with regard to methodological characteristics, interventions and study outcomes using standardized forms. The main data collected was related to the assessment procedures of CAS. Results: Most studies (14 of the 21 included) made an association between the assessment of motor and/or articulatory and segmental skills. Five performed an evaluation of all listed aspects: motor and/or articulatory, segmental and suprasegmental; and two underwent only motor and/or articulatory assessment. The age of the subjects in the present study ranged from 3 to 12 years. Conclusion: The assessment of CAS generally involves the association between the assessment of motor and/or articulatory and segmental skills. It is suggested that further studies in order to evidence validity for the assessment of CAS.
\end{abstract}

Keywords: Rehabilitation of speech and language disorders; Speech; Speech intelligibility; Apraxias; Child

Trabalho realizado no Departamento de Fonoaudiologia, Centro de Ciências da Saúde, Universidade Federal de Santa Catarina - UFSC - Florianópolis (SC), Brasil.

${ }^{1}$ Departamento de Fonoaudiologia, Centro de Ciências da Saúde, Universidade Federal de Santa Catarina - UFSC - Florianópolis (SC), Brasil.

${ }^{2}$ Fonoaudióloga, Florianópolis (SC), Brasil.

Conflito de interesses: Não.

Contribuição dos autores: AMO, IN, GSC e LGG participaram da idealização do estudo, coleta, análise e interpretação dos dados e redação do artigo; AMO e LGG participaram, na condição de orientadoras, da idealização do estudo, análise, interpretação dos dados e redação do artigo.

Autor correspondente: Aline Mara de Oliveira. E-mail: aline.mara.oliveira@ufsc.br

Recebido: Maio 21, 2021; Aceito: Setembro 13, 2021 


\section{INTRODUÇÃO}

A apraxia de fala infantil (AFI) é um distúrbio raro que afeta $0,1 \%$ da população, manifestando-se pela perturbação da habilidade de produzir fonemas e sílabas com precisão e consistência, considerando-se aspectos articulatórios e suprassegmentais. Acredita-se que o planejamento motor deficitário seja o responsável e a base desse transtorno, comprometendo, assim, a formação de palavras e sentenças. Em vista disso, a criança apresenta dificuldades para planejar, de maneira eficaz, a sequência de atos motores necessários para a fala, uma vez que tal tarefa exige movimentos orofaciais rápidos e precisos ${ }^{(1)}$.

Em conformidade com a American Speech-Language-Hearing Association $^{(2)}$, a AFI é um distúrbio de origem neurobiológica, caracterizado por prejudicar, na ausência de comprometimento neuromuscular, a expressão da linguagem em sua modalidade oral, por conta da presença de déficit na precisão e na consistência dos movimentos articulatórios. Ademais, caracteriza-se pela ininteligibilidade de fala, devido à presença de erros durante produções repetitivas de sílabas e palavras, que podem ocorrer tanto em consoantes, como em vogais, com destaque para coarticulação inadequada na transição entre fonemas e sílabas e prosódia inapropriada, principalmente no que diz respeito ao acento lexical e frasal, bem como assistematicidade de erros.

Ainda, a AFI pode advir de comprometimentos no sistema nervoso central, genéticos e/ou somando-se aos distúrbios neurocomportamentais complexos. Ressalta-se, também, que algumas das características da AFI, mencionadas acima, podem estar manifestas em quadros cujos sons da fala estão prejudicados, como em distúrbios de ordem fonológica severos. Fundamentando-se no supracitado, reforça-se a necessidade de uma avaliação minuciosa e embasada, que busque, inclusive, identificar o grau do comprometimento apresentado pelo paciente $^{(3)}$.

Estudos anteriores ${ }^{(4-6)}$ envolveram aspectos motores e/ ou articulatórios, aspectos segmentais das consoantes/vogais e aspectos suprassegmentais no processo de diagnóstico diferencial entre AFI e outras alterações dos distúrbios dos sons da fala (DSF). Especialmente, no que se refere à AFI, é fundamental investigar a presença de inconsistência dos erros (diferentes erros para o mesmo som-alvo) e de interrupções ou alongamentos na transição dos sons (coarticulatória) durante a produção de vogais e/ou consoantes nas sílabas e/ou palavras, como também a realização de padrões prosódicos não esperados (lexical ou frasal) $^{(2,5)}$.

Divergências em relação aos critérios diagnósticos desse transtorno ainda existem, fazendo com que sua caracterização seja complexa ${ }^{(7)}$. A avaliação e o diagnóstico preciso da AFI são discutidos na literatura há anos e, na tentativa de desenvolver um protocolo consistente, alguns instrumentos foram produzidos nas últimas décadas, com vistas a aprimorar o diagnóstico ${ }^{(8)}$.

No final da década de 1990 e no início dos anos 2000, o número de protocolos específicos para a AFI aumentou consideravelmente, com destaque para o Kaufman Speech Praxis Test for Children (KSPT) ${ }^{(9)}$, de 1995 e a Verbal Motor Production Assessment for Children (VMPAC) ${ }^{(10)}$, de 1999. $\mathrm{O}$ KSPT analisa as estruturas orais e a função motora da fala em crianças de 2 a 6 anos $^{(9)}$, ao passo que o VMPAC avalia aspectos do controle oromotor e características da fala na faixa etária de 3 a $12 \operatorname{anos}^{(10)}$. Ambos apresentam evidências de validade de conteúdo e de critério.
Já em 2013, foi publicado um protocolo comumente utilizado na atualidade, o Dynamic Evaluation Motor of Speech Skills (DEMSS) ${ }^{(11)}$, que avalia a função motora, a prosódia e a consistência da produção, diagnosticando desordens dos sons da fala, como a AFI. O teste vem sendo amplamente utilizado em pesquisas e na prática clínica, em razão das evidências de validade e fidedignidade que apresenta ${ }^{(11)}$. No ano de 2016, o DEMSS foi traduzido e adaptado para o português brasileiro (DEMSS-BR) e apresentou evidências de fidedignidade e precisão, porém, ainda se faz necessária a sua validação, bem como a definição de dados normativos ${ }^{(7)}$.

Recentemente, Oliveira et al. ${ }^{(6)}$ propuseram avaliações específicas da produção de fala, vislumbrando realizar o diagnóstico diferencial entre crianças com desvio fonológico severo e crianças com suspeita de AFI. Os autores adaptaram para o português brasileiro, culturalmente e linguisticamente, os seguintes testes: Avaliação de Repetição de Palavras Multissilábicas ${ }^{(12)}$; Avaliação do Acento Frasal ${ }^{(13)}$; Tarefa de Inconsistência de fala ${ }^{(14,15)}$ e Tarefa de Máximo Desempenho ${ }^{(16)}$. Todos os testes mostraram-se sensíveis para diferenciar os grupos de crianças com distúrbios dos sons da fala.

Todavia, ainda que se tenha observado algum crescimento em relação à quantidade de protocolos e suas propriedades psicométricas ao longo dos anos, os parâmetros de avaliação da AFI ainda são, de certa forma, subjetivos e o diagnóstico, por vezes, dá-se pela exclusão de outros comprometimentos ${ }^{(1)}$. Por conseguinte, em âmbito nacional, ainda são escassos os instrumentos para a avaliação da AFI validados e padronizados para a realidade sociocultural do país, o que dificulta o diagnóstico preciso do transtorno ${ }^{(7)}$.

\section{OBJETIVOS}

Considerando o exposto, o presente estudo teve por objetivo revisar sistematicamente os protocolos e/ou avaliações para AFI e classificá-los de acordo com a dimensão clínica avaliada.

\section{ESTRATÉGIAS DE PESQUISA}

Foram pesquisadas as bases de dados eletrônicas (até maio de 2019): MEDLINE (acessado via PubMed), LILACS, Scopus e SciELO. A revisão sistemática foi conduzida conforme as recomendações do PRISMA (Preferred Reporting Items for Systematic reviews and Meta-Analyses). Os descritores foram selecionados no DECs (Descritores em Ciências da Saúde), bem como os operadores booleanos. Os termos de busca usados foram: Apraxias, Childhood apraxia of speech, Evaluation, Assessment, Validation Studies, Evaluation Studies, Language Therapy, Rehabilitation of Speech and Language Disorders e Child, Preschool e seus entretermos. Não foram incluídas palavras relacionadas aos desfechos, para aumentar a sensibilidade desta pesquisa. Não houve restrição quanto ao tipo de avaliação pesquisada, quanto à língua, nem quanto ao delineamento do estudo pesquisado. 


\section{Critérios de seleção}

Foram incluídos todos os estudos cuja definição da AFI mostrava-se completa e embasada cientificamente, bem como aqueles que avaliavam, de forma consistente, sujeitos com suspeita ou diagnóstico confirmado de AFI. Foram excluídos trabalhos cujas amostras não estivessem dentro da faixa etária de zero a 12 anos.

\section{Análise dos dados}

Títulos e resumos de todos os artigos identificados pela estratégia de busca foram avaliados pelos investigadores. Todos os resumos que não apresentavam informações suficientes quanto aos critérios de inclusão e exclusão foram selecionados para avaliação do texto integral. No estágio do texto integral, três revisores, de forma independente, avaliaram os artigos completos e realizaram suas seleções de acordo com os critérios de elegibilidade previamente estipulados. Dois avaliadores independentes realizaram a coleta de dados no que diz respeito às características metodológicas, intervenções e desfechos dos estudos. Foram utilizadas planilhas previamente formatadas para a coleta dos dados. Em todas as etapas do estudo, as discordâncias foram resolvidas por consenso. $\mathrm{O}$ dado principal coletado relacionou-se aos procedimentos de avaliação da AFI para crianças. Para o presente estudo, foram elencadas três dimensões de avaliação da fala, considerando-se: 1) aspectos motores e/ou articulatórios, 2) aspectos segmentais, 3) aspectos suprassegmentais ${ }^{(5)}$.

\section{RESULTADOS}

Como resultado da busca inicial, foram identificados 230 resumos, a partir dos quais 49 estudos atendiam aos critérios de inclusão e foram considerados como potencialmente relevantes para análise posterior detalhada. Após a leitura dos textos integrais, ao total, foram selecionados 20 estudos para compor a amostra desta revisão. A Figura 1 apresenta o diagrama de seleção dos estudos em todas as suas etapas.

A idade dos sujeitos incluídos nos artigos integrantes do presente estudo variou de 3 a 12 anos. As características principais dos estudos incluídos encontram-se na Quadro 1, como autores e ano de publicação, periódico publicado e fator de impacto, delineamento do estudo, número e tipo amostral e instrumentos utilizados.

Ao todo, 19 instrumentos foram utilizados pelos estudos incluídos nesta pesquisa para avaliar a AFI. Dentre eles, 4 mostraram-se os mais frequentes: Goldman-Fristoe Test of Articulation-Second Edition ${ }^{(41)}$ e Diagnostic Evaluation of Articulation and Phonology-DEAP ${ }^{(42)}$, ambos usados em 55\% dos estudos selecionados; Test of Polysyllables ${ }^{(43)}$, citado em $30 \%$ dos estudos incluídos e, ainda, Oral and Speech Motor Control Protocol ${ }^{(44)}$, utilizado por $25 \%$ dos estudos selecionados.

Quanto às dimensões de avaliação, observou-se que, dos 20 estudos incluídos, 14 $4^{(5,19-24,27,28,30,33,34,36,39)}(70 \%)$ realizaram a associação entre a avaliação de habilidades motoras e/ou articulatórias e segmentais. Deste modo, verificou-se que a apraxia de fala na infância é mais comumente avaliada a partir

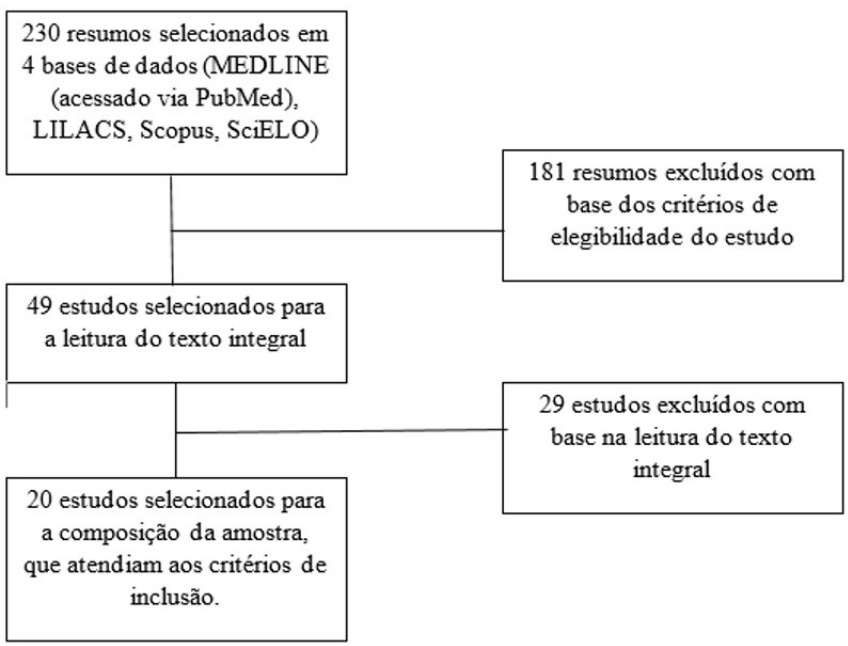

Figura 1. Diagrama de seleção dos estudos

da associação da análise de aspectos articulatórios e/ou motores e segmentais da fala das crianças.

Dos 6 estudos restantes, $5^{(11,17,25,37,38)}(25 \%)$ realizaram avaliação de todos os aspectos elencados pela presente revisão (motor e/ou articulatório, segmental e suprassegmental) e $1^{(18)}$ $(5 \%)$ realizou apenas a avaliação motora e/ou articulatória.

Os instrumentos utilizados para avaliação dos aspectos motores e/ou articulatórios foram: Oral and Speech Motor Control Protocol(44); Goldman-Fristoe Test of ArticulationSecond Edition ${ }^{(41)}$; Verbal Motor Production Assessment for Children - VMPAC ${ }^{(10)}$ e Arabic Articulation Test ${ }^{(29)}$, como demonstra a Quadro 2.

Os protocolos usados para avaliar os aspectos segmentais da fala foram 8: Test of Polysyllables ${ }^{(43)}$; Children's Test of Nonword Repetition - CNRep ${ }^{(46)}$; Syllable Repetition Task ${ }^{(47)}$; The Arabic Syllable Accuracy Word Task ${ }^{(40)}$; Beginner's Intelligibility Test-BIT ${ }^{32)}$; Intelligibility Test of Children's Speech-TOCS ${ }^{(35)}$; Children's Speech Intelligibility Measure-CSIM(11) e, ainda, Maximum Performance Task ${ }^{(48)}$, como demonstra a Quadro 3.

Dois instrumentos foram utilizados para avaliar exclusivamente os aspectos suprassegmentais da fala: Emphatic Stress Task ${ }^{(13)}$ e Profiling Elements of Prosody in Speech-CommunicationPEPS-C ${ }^{(49)}$. Adicionalmente, observou-se que a fala espontânea também pode ser usada como amostra para investigação dos aspectos suprassegmentais da fala, como mostra a Quadro 4.

Destaca-se, também, que alguns testes avaliaram mais de uma dimensão, como o Diagnostic Evaluation of Articulation and Phonology-DEAP ${ }^{(42)}$ e o Kaufman Speech Praxis Test for Children $^{(9)}$, que levaram em conta tanto aspectos motores e/ou articulatórios, quanto aspectos segmentais. Já outros 3 protocolos analisaram todas as três dimensões elencadas neste estudo: Dynamic Evaluation of Motor Speech Skills (DEMSS) ${ }^{(11)} \mathrm{e}$ sua adaptação para o português brasileiro ${ }^{(50)}$; Multisyllabic Word ${ }^{(12)}$ e Strand's 10-point Checklist ${ }^{(51)}$.

\section{DISCUSSÃO}

O presente estudo evidenciou as principais metodologias de avaliação da apraxia de fala na infância, considerando estudos que envolviam crianças de zero a 12 anos. Observou-se, nos estudos incluídos, que a avaliação ocorre de forma mais frequente 
Quadro 1. Características dos estudos incluídos

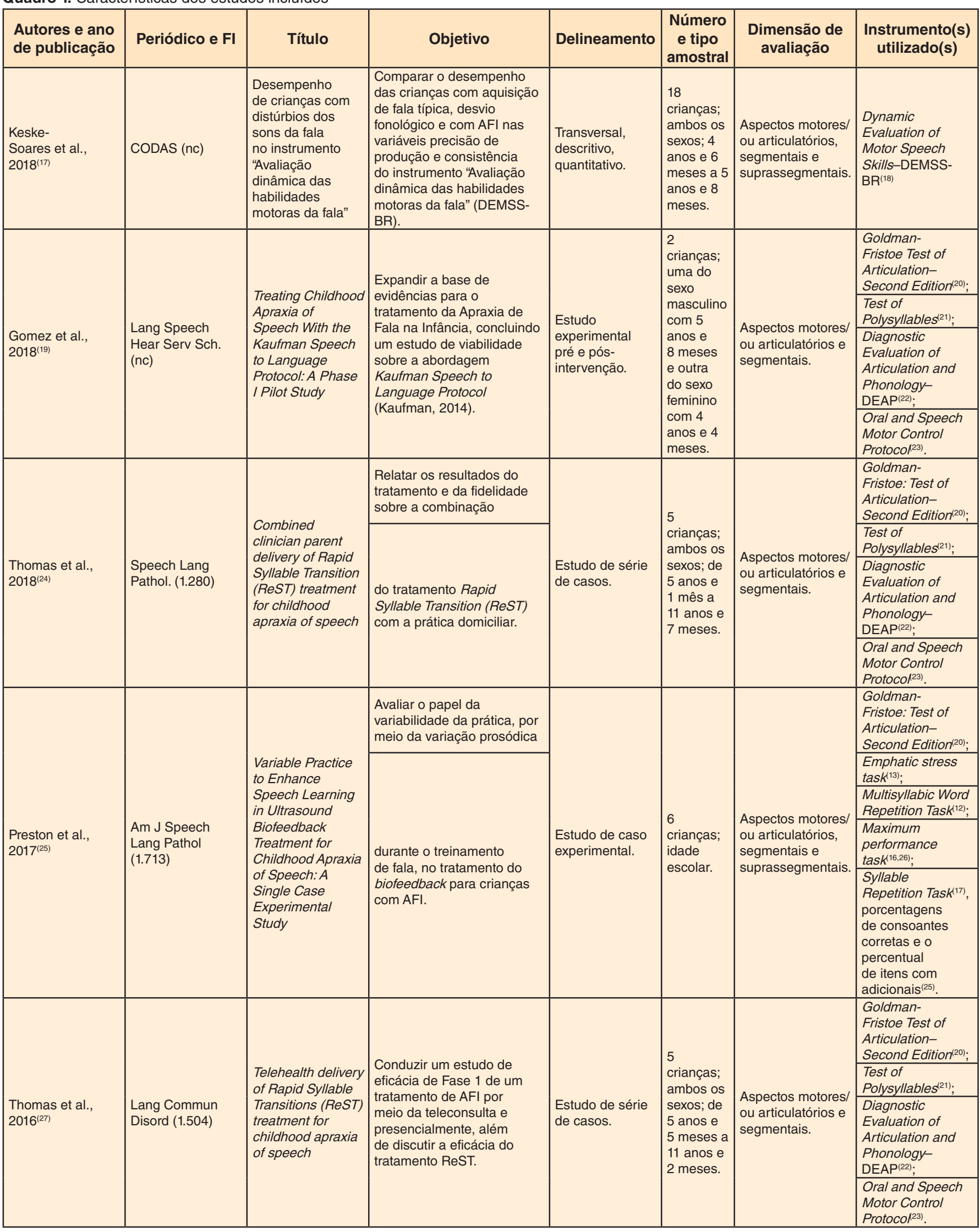

Legenda: $\mathrm{FI}$ = fator de impacto; $\mathrm{nc}=$ não consta $\mathrm{PROMPT}=$ Prompts for Restructuring Oral Muscular Phonetic Targets 
Quadro 1. Continuação...

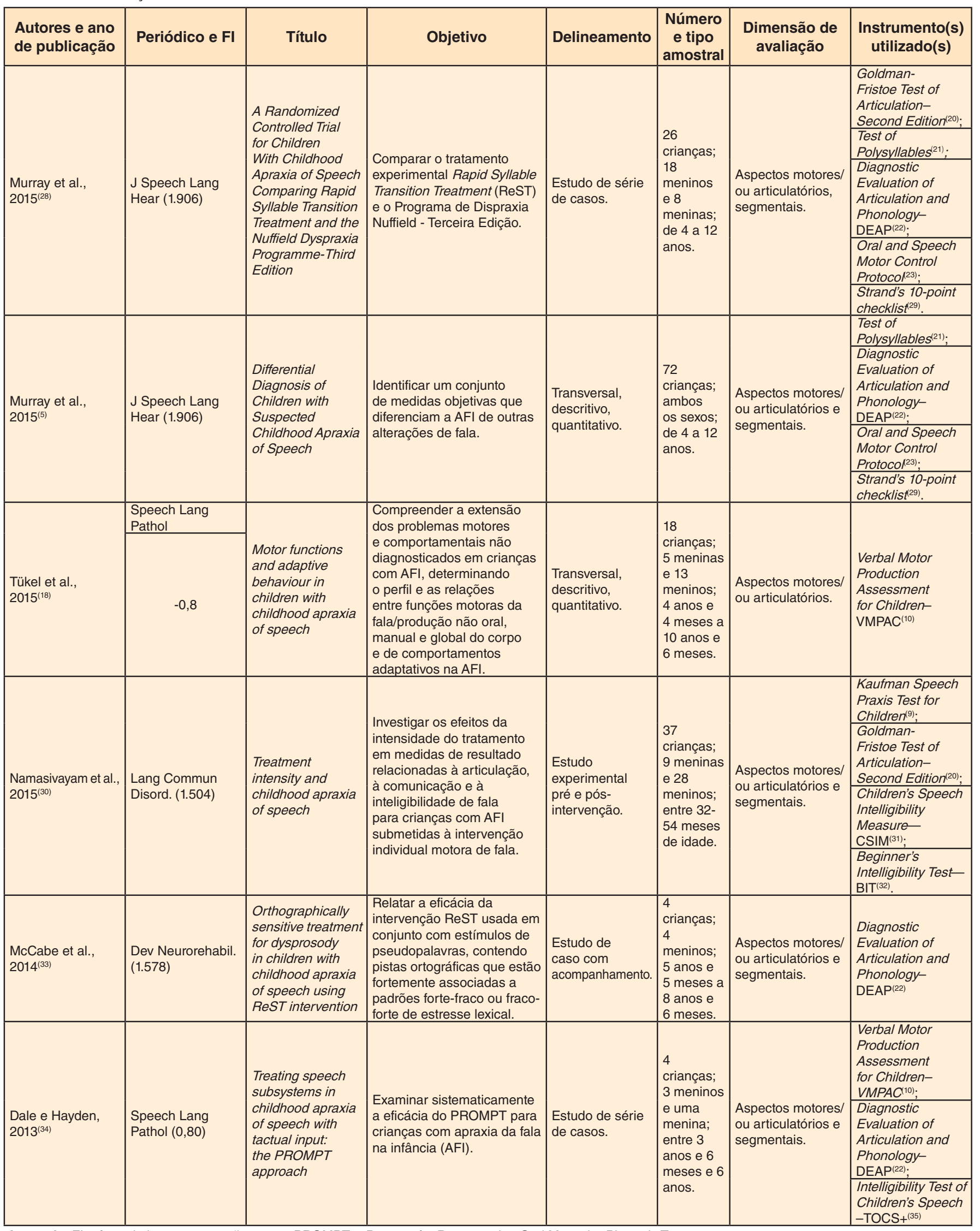

Legenda: $\mathrm{FI}=$ fator de impacto; $\mathrm{nc}=$ não consta PROMPT = Prompts for Restructuring Oral Muscular Phonetic Targets 
Quadro 1. Continuação...

\begin{tabular}{|c|c|c|c|c|c|c|c|}
\hline $\begin{array}{l}\text { Autores e ano } \\
\text { de publicação }\end{array}$ & Periódico e FI & Título & Objetivo & Delineamento & \begin{tabular}{|c|} 
Número \\
e tipo \\
amostral
\end{tabular} & $\begin{array}{l}\text { Dimensão de } \\
\text { avaliação }\end{array}$ & $\begin{array}{c}\text { Instrumento(s) } \\
\text { utilizado(s) }\end{array}$ \\
\hline $\begin{array}{l}\text { Strand et al., } \\
2013^{(11)}\end{array}$ & $\begin{array}{l}\text { Speech Lang } \\
\text { Hear }\end{array}$ & $\begin{array}{l}\text { A motor speech } \\
\text { assessment for } \\
\text { children with } \\
\text { severe speech } \\
\text { disorders: } \\
\text { reliability and } \\
\text { validity evidence }\end{array}$ & $\begin{array}{l}\text { Relatar evidências de } \\
\text { confiabilidade e validade } \\
\text { para a Dynamic Evaluation } \\
\text { of Motor Speech Skills } \\
\text { (DEMSS). }\end{array}$ & $\begin{array}{l}\text { Estudo de } \\
\text { validaçãa. }\end{array}$ & \begin{tabular}{|l|}
81 \\
crianças; \\
63 \\
meninos \\
e 18 \\
meninas; \\
36 a 79 \\
meses de \\
idade. \\
\end{tabular} & $\begin{array}{l}\text { Aspectos motores } \\
\text { e/ou articulatórios, } \\
\text { segmentais e } \\
\text { suprassegmentais. }\end{array}$ & $\begin{array}{l}\text { Dynamic } \\
\text { Evaluation Motor } \\
\text { of Speech Skills- } \\
\text { DEMSS }\end{array}$ \\
\hline \multirow{3}{*}{$\begin{array}{l}\text { Preston et al., } \\
2013^{(36)}\end{array}$} & \multirow{3}{*}{$\begin{array}{l}\text { Speech Lang } \\
\text { Pathol }(0,80)\end{array}$} & \multirow{3}{*}{$\begin{array}{l}\text { Ultrasound } \\
\text { biofeedback } \\
\text { treatment for } \\
\text { persisting } \\
\text { childhood apraxia } \\
\text { of speech }\end{array}$} & \begin{tabular}{|l|} 
Avaliar a eficácia de um \\
programa de tratamento de \\
crianças com persistência \\
de erros dos sons da \\
fala - inclui biofeedback \\
ultrassonográfica - \\
\end{tabular} & \multirow{3}{*}{$\begin{array}{l}\text { Estudo de série } \\
\text { de casos. }\end{array}$} & \multirow{3}{*}{$\begin{array}{l}6 \\
\text { crianças; } \\
\text { todas } \\
\text { do sexo } \\
\text { masculino; } \\
9 \text { a } 10 \\
\text { anos. }\end{array}$} & \multirow{3}{*}{$\begin{array}{l}\text { Aspectos motores/ } \\
\text { ou articulatórios e } \\
\text { segmentais. }\end{array}$} & $\begin{array}{l}\text { Verbal Motor } \\
\text { Production } \\
\text { Assessment } \\
\text { for Children- } \\
\text { VMPAC }^{(10)} \text {; } \\
\end{array}$ \\
\hline & & & associados à AFI. & & & & \begin{tabular}{|l|} 
Goldman- \\
Fristoe Test of \\
Articulation- \\
Second Edition'(20); \\
Diagnostic \\
Evaluation of \\
Articulation and \\
Phonology- \\
DEAP(22); \\
\end{tabular} \\
\hline & & & & & & & $\begin{array}{l}\text { Emphatic Stress } \\
\text { Task }{ }^{131} \text {. }\end{array}$ \\
\hline \multirow{2}{*}{$\begin{array}{l}\text { Maas et al., } \\
2012^{(37)}\end{array}$} & \multirow{2}{*}{$\begin{array}{l}\text { Speech Lang } \\
\text { Pathol (1.906) }\end{array}$} & \multirow{2}{*}{$\begin{array}{l}\text { Feedback } \\
\text { frequency in } \\
\text { treatment for } \\
\text { childhood apraxia } \\
\text { of speech }\end{array}$} & \multirow{2}{*}{$\begin{array}{l}\text { Examinar o papel da } \\
\text { frequência do feedback no } \\
\text { tratamento da AFI. }\end{array}$} & \multirow{2}{*}{$\begin{array}{l}\text { Estudo de série } \\
\text { de casos }\end{array}$} & \multirow{2}{*}{\begin{tabular}{|l|}
4 \\
crianças; \\
2 meninos \\
e 2 \\
meninas; \\
5 a 8 \\
anos de \\
idade. \\
\end{tabular}} & \multirow{2}{*}{$\begin{array}{l}\text { Aspectos motores/ } \\
\text { ou articulatórios, } \\
\text { segmentais e } \\
\text { suprassegmentais. }\end{array}$} & $\begin{array}{l}\text { Dynamic } \\
\text { Evaluation Motor } \\
\text { of Speech Skills- } \\
\text { DEMSS }{ }^{(11)} \text {; } \\
\end{array}$ \\
\hline & & & & & & & $\begin{array}{l}\text { Goldman- } \\
\text { Fristoe Test of } \\
\text { Articulation- } \\
\text { Second Edition }{ }^{(20)} \text {; }\end{array}$ \\
\hline \multirow{2}{*}{$\begin{array}{l}\text { Murray et al., } \\
2012^{(38)}\end{array}$} & \multirow{2}{*}{$\begin{array}{l}\text { BMC Pediatr. } \\
(1.983)\end{array}$} & \multirow{2}{*}{$\begin{array}{l}\text { A comparison of } \\
\text { two treatments } \\
\text { for childhood } \\
\text { apraxia of speech: } \\
\text { methods and } \\
\text { treatment protocol } \\
\text { for a parallel } \\
\text { group randomised } \\
\text { control trial }\end{array}$} & \multirow{2}{*}{$\begin{array}{l}\text { Conduzir um ensaio } \\
\text { clínico em maior escala do } \\
\text { tratamento Rapid Syllable } \\
\text { Transition Treatment (ReST) } \\
\text { comparando com o Nuffield } \\
\text { Dyspraxia Programme - } \\
\text { Third Edition (NDP3). }\end{array}$} & \multirow{2}{*}{$\begin{array}{l}\text { Estudo } \\
\text { controlado } \\
\text { randomizado. }\end{array}$} & \multirow{2}{*}{$\begin{array}{l}20 \\
\text { crianças; } \\
\text { nc; entre } \\
4 \text { e } 12 \\
\text { anos. }\end{array}$} & \multirow{2}{*}{$\begin{array}{l}\text { Aspectos motores/ } \\
\text { ou articulatórios, } \\
\text { segmentais e } \\
\text { suprassegmentais. }\end{array}$} & \begin{tabular}{|l} 
Diagnostic \\
Evaluation of \\
Articulation and \\
Phonology- \\
DEAP(2);
\end{tabular} \\
\hline & & & & & & & $\begin{array}{l}\text { Goldman- } \\
\text { Fristoe Test of } \\
\text { Articulation- } \\
\text { Second Edition } \\
20)\end{array}$ \\
\hline \multirow[t]{2}{*}{$\begin{array}{l}\text { Ballard et al., } \\
2010^{(23)}\end{array}$} & \multirow[t]{2}{*}{$\begin{array}{l}\text { Speech Lang } \\
\text { Hear (1.906) }\end{array}$} & \multirow[t]{2}{*}{$\begin{array}{l}\text { A treatment for } \\
\text { dysprosody in } \\
\text { childhood apraxia } \\
\text { of speech }\end{array}$} & \multirow{2}{*}{$\begin{array}{l}\text { Estudo de Fase II, com } \\
3 \text { crianças. Investigar a } \\
\text { eficácia de um tratamento } \\
\text { que visa melhorar o controle } \\
\text { das durações relativas das } \\
\text { sílabas de não palavras, } \\
\text { composto por } 3 \text { sílabas que } \\
\text { representam padrões de } \\
\text { ênfase forte-fraco e fraco- } \\
\text { forte. }\end{array}$} & \multirow[t]{2}{*}{$\begin{array}{l}\text { Estudo de série } \\
\text { de casos. }\end{array}$} & \multirow{2}{*}{$\begin{array}{l}3 \\
\text { crianças; } \\
2 \text { meninos } \\
\text { e } 1 \\
\text { menina; } \\
\text { entre } 7 \mathrm{e} \\
10 \text { anos. }\end{array}$} & \multirow[t]{2}{*}{$\begin{array}{l}\text { Aspectos motores/ } \\
\text { ou articulatórios e } \\
\text { segmentais. }\end{array}$} & $\begin{array}{l}\text { Goldman- } \\
\text { Fristoe Test of } \\
\text { Articulation- } \\
\text { Second Edition'(20); } \\
\text { Children's Test } \\
\text { of Nonword } \\
\text { Repetition- } \\
\text { CNRep }{ }^{(3) ;} \\
\end{array}$ \\
\hline & & & & & & & $\begin{array}{l}\text { Diagnostic } \\
\text { Evaluation of } \\
\text { Articulation and } \\
\text { Phonology- } \\
\text { DEAP(22). }\end{array}$ \\
\hline \multirow[b]{2}{*}{$\begin{array}{l}\text { luzzini e Forrest, } \\
2010^{(20)}\end{array}$} & \multirow[b]{2}{*}{$\begin{array}{l}\text { Clin Linguist Phon } \\
\text { (nc) }\end{array}$} & \multirow{2}{*}{$\begin{array}{l}\text { Evaluation of } \\
\text { a combined } \\
\text { treatment } \\
\text { approach for } \\
\text { childhood apraxia } \\
\text { of speech }\end{array}$} & \multirow{2}{*}{$\begin{array}{l}\text { Investigar o impacto } \\
\text { de uma abordagem de } \\
\text { tratamento duplo que inclui } \\
\text { protocolo de treinamento } \\
\text { de estimulabilidade } \\
\text { emparelhado com um } \\
\text { tratamento de vocabulário } \\
\text { básico modificado voltado } \\
\text { para os sons da fala } \\
\text { produzidos por crianças } \\
\text { com AFI. }\end{array}$} & \multirow[b]{2}{*}{ Estudo de caso. } & \multirow{2}{*}{$\begin{array}{l}4 \\
\text { crianças; } \\
2 \text { meninos } \\
\text { e } 2 \\
\text { meninas; } \\
\text { entre } 3 \text { e } \\
6 \text { anos. }\end{array}$} & \multirow[b]{2}{*}{$\begin{array}{l}\text { Aspectos motores/ } \\
\text { ou articulatórios e } \\
\text { segmentais. }\end{array}$} & $\begin{array}{l}\text { Goldman- } \\
\text { Fristoe Test of } \\
\text { Articulation- } \\
\text { Second Edition } \\
\end{array}$ \\
\hline & & & & & & & $\begin{array}{l}\text { Diagnostic } \\
\text { Evaluation of } \\
\text { Articulation and } \\
\text { Phonology- } \\
\text { DEAP(22). }\end{array}$ \\
\hline
\end{tabular}

Legenda: $\mathrm{FI}$ = fator de impacto; $\mathrm{nc}=$ não consta PROMPT = Prompts for Restructuring Oral Muscular Phonetic Targets 
Quadro 1. Continuação...

\begin{tabular}{|c|c|c|c|c|c|c|c|}
\hline $\begin{array}{l}\text { Autores e ano } \\
\text { de publicação }\end{array}$ & Periódico e FI & Título & Objetivo & Delineamento & \begin{tabular}{|c|} 
Número \\
e tipo \\
amostral \\
\end{tabular} & $\begin{array}{l}\text { Dimensão de } \\
\text { avaliação }\end{array}$ & $\begin{array}{c}\text { Instrumento(s) } \\
\text { utilizado(s) }\end{array}$ \\
\hline \multirow[b]{2}{*}{ Aziz et al., 2010(22) } & \multirow[b]{2}{*}{$\begin{array}{l}\text { J Pediatr } \\
\text { Otorhinolaryngol. } \\
\text { (1.125) }\end{array}$} & \multirow{2}{*}{$\begin{array}{l}\text { Childhood } \\
\text { apraxia of speech } \\
\text { and multiple } \\
\text { phonological } \\
\text { disorders in Cairo- } \\
\text { Egyptian Arabic } \\
\text { speaking children: } \\
\text { Language, } \\
\text { speech, and oro- } \\
\text { motor differences }\end{array}$} & \multirow{2}{*}{$\begin{array}{l}\text { Questionar uma possível } \\
\text { diferença significativa } \\
\text { no desempenho oral de } \\
\text { linguagem, fala e não } \\
\text { fala entre crianças com } \\
\text { apraxia de fala na infância, } \\
\text { com transtorno fonológico } \\
\text { múltiplo e crianças típicas } \\
\text { que podem ser utilizadas } \\
\text { para fins de diagnósticos } \\
\text { diferenciais. }\end{array}$} & \multirow[b]{2}{*}{$\begin{array}{l}\text { Estudo de caso- } \\
\text { controle. }\end{array}$} & \multirow[b]{2}{*}{$\begin{array}{l}30 \\
\text { crianças; } \\
16 \\
\text { meninos } \\
\text { e 14 } \\
\text { meninas; } \\
\text { entre 4 e } \\
6 \text { anos. }\end{array}$} & \multirow[b]{2}{*}{$\begin{array}{l}\text { Aspectos motores/ } \\
\text { ou articulatórios e } \\
\text { segmentais. }\end{array}$} & $\begin{array}{l}\text { Arabic Articulation } \\
\text { Test }^{29}\end{array}$ \\
\hline & & & & & & & $\begin{array}{l}\text { The Arabic } \\
\text { Syllable Accuracy } \\
\text { Word Task } \\
\text { (ASAWT) }^{(40)}\end{array}$ \\
\hline $\begin{array}{l}\text { Sealey e Giddens, } \\
2010^{(21)}\end{array}$ & $\begin{array}{l}\text { Clin Linguist Phon } \\
\text { (nc) }\end{array}$ & $\begin{array}{l}\text { Aerodynamic } \\
\text { indices of } \\
\text { velopharyngeal } \\
\text { function in } \\
\text { childhood apraxia } \\
\text { of speech } \\
\end{array}$ & $\begin{array}{l}\text { Documentar as diferenças } \\
\text { na função velofaríngea em } \\
\text { crianças com diagnóstico } \\
\text { de AFI e de crianças com } \\
\text { desenvolvimento de fala } \\
\text { típico. }\end{array}$ & $\begin{array}{l}\text { Estudo de caso- } \\
\text { controle }\end{array}$ & $\begin{array}{l}6 \\
\text { crianças; } \\
\text { nc; entre } 5 \\
\text { e } 9 \text { anos. }\end{array}$ & $\begin{array}{l}\text { Aspectos motores/ } \\
\text { ou articulatórios e } \\
\text { segmentais. }\end{array}$ & $\begin{array}{l}\text { Kaufman Speech } \\
\text { Praxis Test for } \\
\text { Children }{ }^{(9)}\end{array}$ \\
\hline
\end{tabular}

Legenda: $\mathrm{FI}$ = fator de impacto; $\mathrm{nc}=$ não consta PROMPT = Prompts for Restructuring Oral Muscular Phonetic Targets

Quadro 2. Protocolos para avaliação motora e/ou articulatória

\begin{tabular}{|c|c|c|c|c|c|c|}
\hline Autor e Ano & Nome e autores & Público-alvo & O que avalia & Objetivos & Particularidades & $\begin{array}{l}\text { Propriedades } \\
\text { psicométricas }\end{array}$ \\
\hline \multirow{4}{*}{$\begin{array}{l}\text { Gomez et al., } 2018^{(19)} \text {; } \\
\text { Thomas et al., } \\
2018^{(24)} \text {; Murray et al., } \\
2015^{(28)} \text {; Murray et al., } \\
2015^{(5)}\end{array}$} & \multirow{4}{*}{$\begin{array}{l}\text { Oral and Speech } \\
\text { Motor Control } \\
\text { Protocol (Robbins e } \\
\text { Klee, 1987) }\end{array}$} & \multirow{4}{*}{$\begin{array}{l}\text { Crianças americanas } \\
\text { de } 2 \text { anos e } 6 \text { meses } \\
\text { a } 6 \text { anos e } 11 \text { meses. }\end{array}$} & \multirow{4}{*}{$\begin{array}{l}\text { Avalia as estruturas e } \\
\text { funções orofaciais. }\end{array}$} & \multirow{4}{*}{$\begin{array}{l}\text { Determinar se os } \\
\text { deficit nas estruturas } \\
\text { ou funções orais } \\
\text { podem explicar as } \\
\text { dificuldades de fala. }\end{array}$} & Contém três partes: & \multirow{4}{*}{$\begin{array}{l}\text { Apresenta medidas } \\
\text { de confiabilidade e } \\
\text { consistência interna } \\
\text { (Robbins e Klee, } \\
\text { 1987)(44) }\end{array}$} \\
\hline & & & & & $\begin{array}{l}\text { 1. Avaliação das } \\
\text { estruturas, feita a } \\
\text { partir de inspeções } \\
\text { visuais; }\end{array}$ & \\
\hline & & & & & $\begin{array}{l}\text { 2. Avaliação } \\
\text { funcional, feita a } \\
\text { partir de comandos } \\
\text { verbais; }\end{array}$ & \\
\hline & & & & & $\begin{array}{l}\text { 3. Avaliação da taxa } \\
\text { de repetição de } \\
\text { sílaba e da duração } \\
\text { do prolongamento da } \\
\text { vogal. } \\
\end{array}$ & \\
\hline \multirow{3}{*}{$\begin{array}{l}\text { Gomez et al., } 2018^{(19)} ; \\
\text { Thomas et al., } \\
2018^{(24)} ; \\
\text { Preston et al., } \\
2017^{(25)} \text {; } \\
\text { Thomas et al., } \\
2016^{(27)} \text {; Murray et al., } \\
2015^{(28)} \text { Ballard et al., } \\
2010^{(23)} \text {; Iuzzini e } \\
\text { Forrest, } 2010^{(20)} \\
\end{array}$} & \begin{tabular}{|l|}
$\begin{array}{l}\text { Goldman-Fristoe Test } \\
\text { of Articulation }\end{array}$ \\
\end{tabular} & \multirow{3}{*}{$\begin{array}{l}\text { Pode ser utilizado } \\
\text { em uma ampla faixa } \\
\text { etária: } 2 \text { anos e } 0 \\
\text { meses a } 21 \text { anos e } \\
11 \text { meses. }\end{array}$} & \multirow{3}{*}{$\begin{array}{l}\text { Avalia a produção } \\
\text { de consoantes em } \\
\text { posição inicial, } \\
\text { medial e final, bem } \\
\text { como a produção } \\
\text { de encontros } \\
\text { consonantais. }\end{array}$} & Medir a articulação & \multirow[b]{3}{*}{$\begin{array}{l}\text { É composto por } \\
34 \text { imagens que } \\
\text { possibilitam obter até } \\
53 \text { palavras-alvo. }\end{array}$} & \multirow{3}{*}{$\begin{array}{l}\text { Validado para a } \\
\text { língua inglesa e } \\
\text { normatizado por } \\
\text { gênero (homem } \\
\text { e mulher) para a } \\
\text { população norte- } \\
\text { americana. }\end{array}$} \\
\hline & \begin{tabular}{|l|} 
GFTA-2 \\
\end{tabular} & & & & & \\
\hline & $\begin{array}{l}\text { Goldman e Fristoe, } \\
2000^{(41)}\end{array}$ & & & $\begin{array}{l}\text { dos sons } \\
\text { consonantais e } \\
\text { determinar os tipos } \\
\text { de erros de produção } \\
\text { de fala. }\end{array}$ & & \\
\hline \multirow{6}{*}{$\begin{array}{l}\text { Tükel et al., } \\
2015^{(18)} \text {; Dale e } \\
\text { Hayden, 2013(34); } \\
\text { Preston et al., } 2013^{(36)}\end{array}$} & \begin{tabular}{|l|} 
Verbal Motor \\
Production \\
Assessment \\
\end{tabular} & \multirow{6}{*}{$\begin{array}{l}\text { Crianças na faixa } \\
\text { etária de } 3 \text { anos a } 12 \\
\text { anos e } 11 \text { meses. }\end{array}$} & \multirow{6}{*}{$\begin{array}{l}\text { Avalia as funções } \\
\text { motoras da fala } \\
\text { e também as } \\
\text { estruturas orais. }\end{array}$} & \multirow{6}{*}{$\begin{array}{l}\text { Analisar a precisão } \\
\text { e a qualidade dos } \\
\text { movimentos motores, } \\
\text { identificando os } \\
\text { níveis de interrupção } \\
\text { motora da fala. }\end{array}$} & \begin{tabular}{|l|} 
É composto por 82 \\
itens, subdivididos \\
em 5 áreas: \\
\end{tabular} & \multirow{6}{*}{$\begin{array}{l}\text { Estudo e } \\
\text { confiabilidade } \\
\text { teste-reteste e entre } \\
\text { examinadores; } \\
\text { validade de conteúdo } \\
\text { e de construto; } \\
\text { normatizado } \\
\text { e padronizado } \\
\text { (McCauley e Strand, } \\
2008)^{(45)}\end{array}$} \\
\hline & \multirow{5}{*}{$\begin{array}{l}\text { for Children- VMPAC } \\
\text { (Hayden e Square, } \\
\text { 1999)(10) }\end{array}$} & & & & $\begin{array}{l}\text { 1. controle motor } \\
\text { global; }\end{array}$ & \\
\hline & & & & & 2. controle oromotor; & \\
\hline & & & & & 3. sequenciamento; & \\
\hline & & & & & $\begin{array}{l}\text { 4. fala encadeada e } \\
\text { linguagem oral; }\end{array}$ & \\
\hline & & & & & $\begin{array}{l}\text { 5. características } \\
\text { da fala. }\end{array}$ & \\
\hline
\end{tabular}


Quadro 2. Continuação...

\begin{tabular}{|c|l|c|c|c|c|c|}
\hline Autor e Ano & Nome e autores & Público-alvo & O que avalia & Objetivos & Particularidades & $\begin{array}{l}\text { Propriedades } \\
\text { psicométricas }\end{array}$ \\
\hline Aziz et al., 2010(22) & $\begin{array}{l}\text { Arabic Articulation } \\
\text { Test (Kotby et al., } \\
1986)^{(29)}\end{array}$ & N.C. & $\begin{array}{l}\text { Avalia a produção } \\
\text { de consoantes e } \\
\text { vogais isoladamente } \\
\text { e também em } \\
\text { diferentes posições } \\
\text { das palavras (inicial, } \\
\text { intermédia e final). }\end{array}$ & $\begin{array}{l}\text { Analisar a produção } \\
\text { da palavra em nível } \\
\text { articulatório. }\end{array}$ & $\begin{array}{l}\text { imagens, que devem } \\
\text { ser nomeadas, } \\
\text { permitindo a } \\
\text { avaliação de 23 } \\
\text { consoantes árabes e } \\
\text { também de 6 vogais. }\end{array}$ & N.C. \\
\hline
\end{tabular}

Legenda: N.C. $=$ Não Consta. Fonte: Elaborada pelos autores

Quadro 3. Protocolos para avaliação segmental da fala

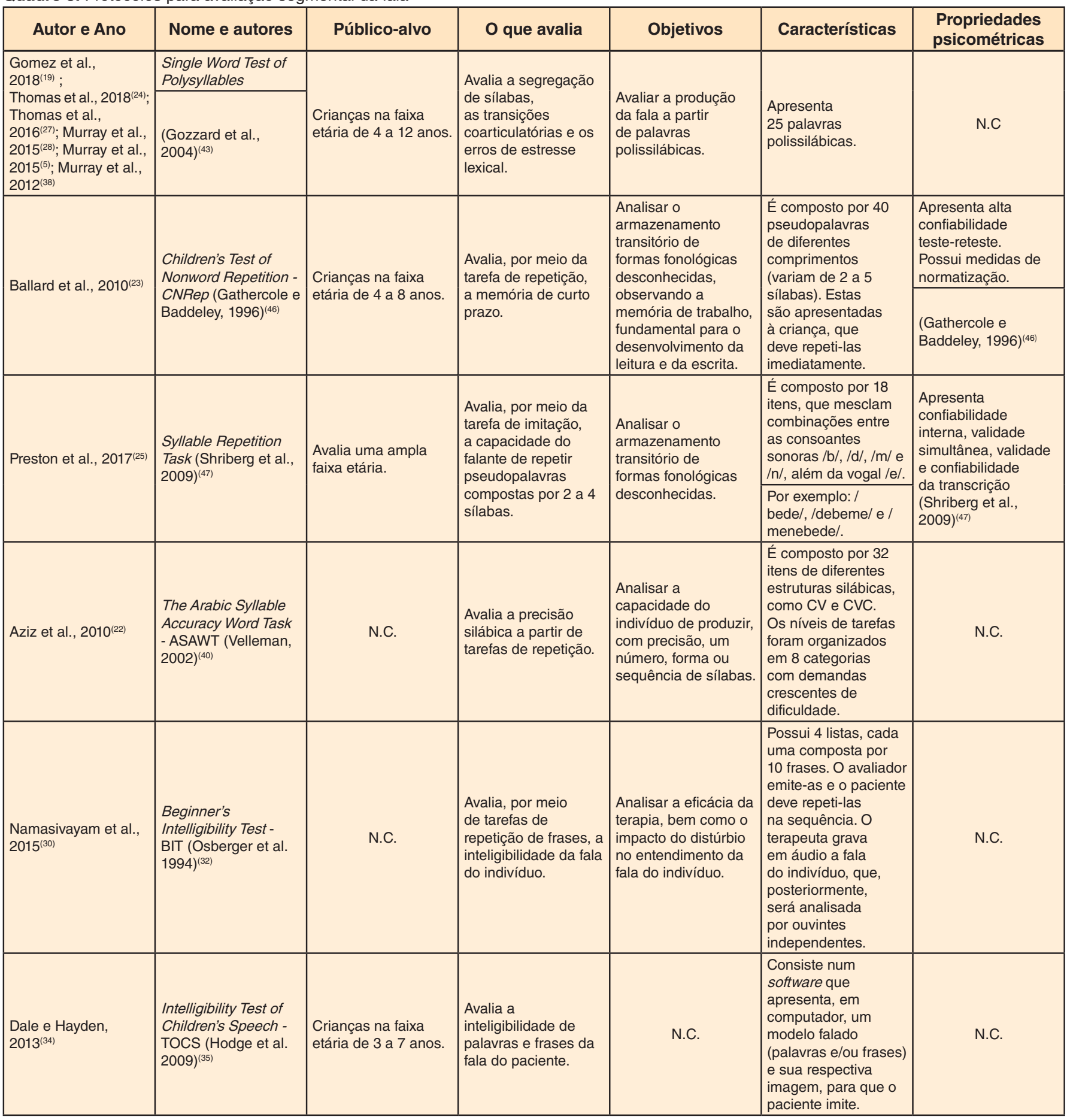


Quadro 3. Continuação...

\begin{tabular}{|c|c|c|c|c|c|c|}
\hline Autor e Ano & Nome e autores & Público-alvo & O que avalia & Objetivos & Características & $\begin{array}{l}\text { Propriedades } \\
\text { psicométricas }\end{array}$ \\
\hline $\begin{array}{l}\text { Wilcox e Morris, } \\
1999^{(31)}\end{array}$ & $\begin{array}{l}\text { Children's Speech } \\
\text { Intelligibility Measure } \\
\text { - CSIM (Wilcox e } \\
\text { Morris, 1999) }{ }^{(31)}\end{array}$ & N.C. & $\begin{array}{l}\text { Avalia, por meio de } \\
\text { tarefas de repetição } \\
\text { de palavras, a } \\
\text { inteligibilidade da fala } \\
\text { do indivíduo. }\end{array}$ & $\begin{array}{l}\text { Analisar a eficácia } \\
\text { da terapia, assim } \\
\text { como o impacto } \\
\text { do distúrbio no } \\
\text { entendimento da fala } \\
\text { do paciente. }\end{array}$ & $\begin{array}{l}\text { É composto por } \\
\text { listas de } 50 \text { palavras, } \\
\text { que se diferem } \\
\text { na avaliação pré } \\
\text { e pós-tratamento. } \\
\text { O paciente deve } \\
\text { imitar o modelo } \\
\text { do terapeuta e } \\
\text { sua fala deve ser } \\
\text { gravada em áudio. } \\
\text { Posteriormente, } \\
\text { este será analisado } \\
\text { por ouvintes } \\
\text { independentes. }\end{array}$ & N.C. \\
\hline $\begin{array}{l}\text { Rvachew et al. } \\
(2005)^{(48)}\end{array}$ & $\begin{array}{l}\text { Maximum } \\
\text { Performance Task } \\
\text { (Rvachew et al., } \text { 2005)(48) }\end{array}$ & N.C. & $\begin{array}{l}\text { Avalia o } \\
\text { funcionamento motor } \\
\text { da fala. }\end{array}$ & $\begin{array}{l}\text { Analisar a duração } \\
\text { da fonação, a rapidez } \\
\text { com que as sílabas } \\
\text { podem ser repetidas } \\
\text { etc. }\end{array}$ & $\begin{array}{l}\text { É composto } \\
\text { por tarefas de } \\
\text { sustentação de } \\
\text { fricativas e de } \\
\text { vogais, bem como de } \\
\text { produção repetida de } \\
\text { monossílabos e do } \\
\text { trissílabo /pataka/. }\end{array}$ & $\begin{array}{l}\text { Normatização; } \\
\text { Validade preditiva: } \\
\text { sensibilidade e } \\
\text { especificidade }^{(16)}\end{array}$ \\
\hline
\end{tabular}

Legenda: N.C. = Não Consta. Fonte: Elaborada pelos autores

Quadro 4. Protocolos para avaliação suprassegmental da fala

\begin{tabular}{|c|c|c|c|c|c|c|}
\hline Autor e Ano & Nome e autores & Público-alvo & O que avalia & Objetivos & Particularidades & $\begin{array}{l}\text { Propriedades } \\
\text { psicométricas }\end{array}$ \\
\hline \multirow{3}{*}{$\begin{array}{l}\text { Shriberg et al., } \\
2010^{(13)}\end{array}$} & Emphatic & \multirow{3}{*}{ N.C. } & \multirow{3}{*}{$\begin{array}{l}\text { Avalia a } \\
\text { capacidade } \\
\text { de imitar } \\
\text { corretamente o } \\
\text { acento frasal. }\end{array}$} & \multirow{3}{*}{$\begin{array}{l}\text { Verificar se a } \\
\text { criança percebe } \\
\text { o contraste entre } \\
\text { as palavras, } \\
\text { diferenciando a } \\
\text { palavra acentuada } \\
\text { daquela não } \\
\text { acentuada. }\end{array}$} & $\begin{array}{l}\text { É composto por } \\
\text { frases, como: } \\
\text { "João ADORA } \\
\text { jogar bola". }\end{array}$ & \multirow{3}{*}{$\begin{array}{l}\text { Foi recentemente } \\
\text { traduzido para } \\
\text { o português } \\
\text { brasileiro } \\
\text { (Oliveira, et al. } \\
2020)^{(6)}\end{array}$} \\
\hline & Stress Task & & & & O avaliador & \\
\hline & $\begin{array}{l}\text { (Shriberg et al., } \\
\text { 2010)(13) }\end{array}$ & & & & $\begin{array}{l}\text { pronuncia a frase } \\
\text { e a criança deve } \\
\text { repeti-la. }\end{array}$ & \\
\hline \multirow[b]{2}{*}{$\begin{array}{l}\text { Peppé e McCann, } \\
(2003)^{(49)}\end{array}$} & $\begin{array}{l}\text { Profiling Elements } \\
\text { of Prosody } \\
\text { in Speech- } \\
\text { Communication- } \\
\text { PEPS-C }\end{array}$ & \multirow[b]{2}{*}{$\begin{array}{l}\text { Ampla faixa etária, } \\
\text { que abrange tanto } \\
\text { adultos quanto } \\
\text { crianças. }\end{array}$} & \multirow[b]{2}{*}{$\begin{array}{l}\text { Avalia as } \\
\text { habilidades } \\
\text { prosódicas } \\
\text { receptivas e } \\
\text { expressivas em } \\
\text { dois níveis, formal } \\
\text { e funcional }\end{array}$} & \multirow[b]{2}{*}{$\begin{array}{l}\text { Avaliar o } \\
\text { comprometimento } \\
\text { prosódico em } \\
\text { indivíduos com } \\
\text { distúrbios de fala e } \\
\text { de linguagem. }\end{array}$} & $\begin{array}{l}\text { Uma expressão } \\
\text { falada é } \\
\text { reproduzida, } \\
\text { enquanto } \\
\text { duas opções }\end{array}$ & \multirow[b]{2}{*}{$\begin{array}{l}\text { O PEPS-C não } \\
\text { é padronizado e } \\
\text { existem apenas } \\
\text { alguns dados } \\
\text { normativos. } \\
\text { Entretanto, já foi } \\
\text { adaptado para } \\
\text { diversas línguas }^{(49)} \text {. }\end{array}$} \\
\hline & $\begin{array}{l}\text { Peppé e McCann } \\
(2003)^{(49)}\end{array}$ & & & & $\begin{array}{l}\text { aparecem na } \\
\text { tela de um } \\
\text { computador. O } \\
\text { paciente deve } \\
\text { escolher a que } \\
\text { corresponde com } \\
\text { a declaração } \\
\text { ouvida, quando a } \\
\text { tarefa é receptiva, } \\
\text { ou falar sobre } \\
\text { as imagens, } \\
\text { quando a tarefa é } \\
\text { expressiva. }\end{array}$ & \\
\hline
\end{tabular}

Legenda: N.C. = Não Consta. Fonte: Elaborada pelos autores

envolvendo a associação entre a avaliação de habilidades motoras e/ou articulatórias e segmentais. Ainda, alguns estudos realizaram apenas avaliação motora e outros, a junção das três dimensões. Verificou-se que a avaliação associada, ou seja, incluindo mais de uma dimensão, favorece a melhor compreensão do desempenho de fala da criança, fornecendo informações mais rebuscadas e aprofundadas sobre a fala e possibilitando melhores condições para a organização de intervenções eficazes.
Esses achados concordam com outros estudos, como a pesquisa brasileira $^{(7)}$ que afirmou que, para um melhor diagnóstico, deve ser realizada uma avaliação combinada a partir da aplicação de diferentes protocolos validados e fidedignos.

Dos 20 estudos incluídos, 14 (5,19-24,27,28,30,33,34,36,39) consideraram, para avaliação da AFI, a associação entre essas habilidades. Verificou-se que os principais fatores avaliados pelos estudos incluídos nesta pesquisa são relacionados às habilidades motoras 
e/ou articulatórias e segmentais. Sendo assim, a forma mais frequente de avaliar a apraxia de fala na infância foi a partir da associação de tais habilidades.

Apenas 24\% ${ }^{(11,17,25,37,38)}$ dos trabalhos selecionados na presente revisão analisaram as habilidades suprassegmentais da fala, fato que pode comprometer o diagnóstico da AFI. Pesquisas ${ }^{(13,17,52)}$ têm afirmado que as características suprassegmentais contribuem na composição da avaliação, favorecendo o diagnóstico diferencial, visto que os indivíduos com AFI frequentemente apresentam prosódia inadequada, em decorrência da inconsistência do acento lexical.

Segundo a American Speech-Language-Hearing Association $(\mathrm{ASHA})^{(2)}$, o diagnóstico de AFI requer que uma criança, no mínimo, atenda a todas as três características, a saber: (1) inconsistência entre palavras e sílabas; (2) transições coarticulatórias alongadas e interrompidas e (3) prosódia inadequada. Dessa forma, verificou-se que a maioria dos estudos não contemplaram os aspectos prosódicos (habilidades suprassegmentais da fala), tanto do ponto de vista do nível frasal, quanto do ponto de vista lexical.

O instrumento Dynamic Evaluation Motor of Speech Skills $(\mathrm{DEMSS})^{(11)}$, bem como sua versão brasileira (DEMSS-BR) ${ }^{(50)}$, destaca-se entre os demais protocolos, pois se mostra bastante completo, tendo em vista que avalia todas as três habilidades: motora e/ou articulatória, segmental e suprassegmental. $\mathrm{O}$ estudo ${ }^{(17)}$, realizado com 18 crianças, concluiu que o protocolo é sensível para diagnosticar a AFI, cumprindo seu propósito de auxiliar no diagnóstico diferencial dos distúrbios dos sons da fala. A pesquisa ${ }^{(17)}$ apontou, ainda, que as variáveis "precisão da produção" e "consistência da fala", contidas no teste, são significativamente pertinentes no processo avaliativo.

Logo, o protocolo $\mathrm{DEMSS}^{(11)}$ deve ser considerado pelo avaliador no momento da escolha do instrumento de avaliação. Vale destacar que o DEMSS-BR está em processo de adaptação e ainda não é validado, porém, vem apresentando precisão, estabilidade e evidências de fidedignidade ${ }^{(50)}$. É indispensável ressaltar que a tradução de protocolos é apenas o primeiro passo do processo, sendo fundamental a realização de adaptações transculturais.

Salienta-se, também, a significativa falta de instrumentos com evidências psicométricas para avaliar a AFI no Brasil. Dentre os protocolos citados nos estudos incluídos nesta pesquisa, pouquíssimos são traduzidos para o português brasileiro e nenhum está adaptado transculturalmente. Destaca-se apenas o já citado DEMSS-BR ${ }^{(50)}$ e a recente tradução da Multisyllabic Word $^{(12)}$ (Avaliação de Repetição de Palavras Multissilábicas) por Oliveira et al. $(2020)^{(6)}$, o que comprova a grande lacuna existente no que diz respeito à avaliação da AFI em âmbito nacional.

Outro estudo ${ }^{(53)}$ reitera, ainda, que a apraxia repercute em todos os níveis linguísticos da criança - sintático, semântico, pragmático, fonético e fonológico, reforçando, portanto, a importância de uma avaliação ampla da linguagem para um diagnóstico preciso e adequado e não apenas de aspectos motores e/ou articulatórios, mesmo que esses se mostrem significativamente comprometidos na apraxia de fala infantil.

Além disso, dos marcadores clínicos propostos pela $\mathrm{ASHA}^{(2)}$ para que um diagnóstico de AFI seja preciso, as crianças precisam de intenção comunicativa, independentemente da idade ou gravidade. Por essas razões, alguns estudos incluídos na presente revisão referem métodos/instrumentos não específicos para AFI, mas que avaliaram a linguagem e a fala de forma mais abrangente, tais como: Peabody Picture Vocabulary Test-Fourth Edition $^{(26)}$ e Clinical Evaluation of Language Fundamentals Preschool-Second Edition ${ }^{(54)}$, incluídos no estudo ${ }^{(19)}$. O Clinical Evaluation of Language Fundamentals-Fourth Edition ${ }^{(54)}$ foi utilizado por diversos estudos ${ }^{(5,20,23,24,37)}$.

Sugere-se que mais estudos sejam realizados com o objetivo de buscar evidências psicométricas voltadas especificamente para avaliação da AFI, incluindo aspectos articulatórios, motores e suprassegmentais da fala. Adicionalmente, é importante que mais revisões como esta sejam realizadas, incluindo estudos com outras faixas etárias. Dessa forma, ampliar estudos que abranjam o processo avaliativo da AFI, inclusive o processo de tradução e adaptação transcultural, que incorporem medidas psicométricas nos diferentes parâmetros da produção de fala (avaliações que contemplem os aspectos motores e/ou articulatórios, segmentais e suprassegmentais, separadamente e associados) é importante, pois tais aspectos compõem os critérios diagnósticos para AFI.

Como limitações da presente revisão, constata-se a não uniformização dos delineamentos das pesquisas incluídas e o reduzido número de estudos brasileiros que envolvam avaliações contemplando todas as habilidades (motoras e/ou articulatórias, segmentais e suprassegmentais) para alcançar o diagnóstico de AFI.

Outra limitação determinante foi a dificuldade de acesso a alguns protocolos de avaliação originais, tais como Kaufman Speech Praxis Test for Children KSPT) $^{(9,19,30)}$, Dynamic Evaluation of Motor Speech Skill(11,18) e Verbal Motor Production Assessment for Children-VMPAC ${ }^{(10,18,34,36)}$. Entretanto, em decorrência da sua importância para o escopo desta pesquisa, optou-se por referenciá-los de modo secundário, ou seja, a partir das descrições de pesquisas que fizeram a sua utilização, sendo possível, assim, sua descrição e caracterização neste estudo.

\section{CONCLUSÃO}

A avaliação da AFI ocorre, de forma mais frequente, envolvendo a associação entre a análise de habilidades motoras e/ou articulatórias e segmentais. Isto posto, constatou-se que a avaliação associada, ou seja, incluindo mais de uma dimensão, favorece a melhor compreensão do desempenho de fala da criança, fornecendo informações mais específicas a respeito do desenvolvimento da fala, o que possibilita a organização de intervenções mais eficazes.

Muitos estudos não incluem a avaliação de aspectos suprassegmentais da fala, demonstrando, assim, uma lacuna significativa na avaliação da AFI em crianças. Observou-se, também, que, no Brasil, são poucos os instrumentos específicos para a AFI, evidenciando a necessidade de mais esforços para adaptar transculturalmente os protocolos já existentes e amplamente utilizados em outros países.

\section{REFERÊNCIAS}

1. Morgan AT, Murray E, Liégeois FJ. Interventions for childhood apraxia of speech. Cochrane Database Syst Rev. 2018;5(5):CD006278. PMid:29845607.

2. ASHA: American-Speech-Language-Hearing Association. Childhood apraxia of speech [Internet]. Rockville: ASHA; 2007 [citado em 2019 
Ago 27]. Disponível em: http://www.asha.org/policy/PS2007-00277. $\mathrm{htm}$

3. Shriberg LD, Wren YE. A frequent acoustic sign of speech motor delay (SMD). Clin Linguist Phon. 2019;33(8):757-71. http://dx.doi. org/10.1080/02699206.2019.1595734. PMid:30945568.

4. Fish M. Here's how to treat childhood apraxia of speech. San Diego, CA: Plural Publishing; 2016.

5. Murray E, McCabe P, Heard R, Ballard K. Differential diagnosis of children with suspected childhood apraxia of speech. J Speech Lang Hear Res. 2015;58(1):43-60. http://dx.doi.org/10.1044/2014 JSLHR-S-12-0358. PMid:25480674.

6. Oliveira AM, Veschi GV, Polii L, Silva CEE, Berti LC. Speech production measures in Brazilian Portuguese children with and without Speech Sound Disorder. In: Babatsouli E, editor. On under-reported monolingual child phonology. 1st ed. Bristol: Multilingual Matters; 2020. p. 380-400.

7. Gubiani MB, Pagliarin KC, Keske-Soares M. Instrumentos para avaliação de apraxia de fala infantil. CoDAS. 2015;27(6):610-5. http:// dx.doi.org/10.1590/2317-1782/20152014152. PMid:26691627.

8. Marini C. Habilidades práxicas orofaciais em crianças com desvio fonológico evolutivo e com desenvolvimento fonológico típico [tese]. Santa Maria: Curso de Distúrbios da Comunicação Humana, Universidade Federal de Santa Maria; 2010.

9. Kaufman N. Kaufman speech praxis test for children. Detroit: Wayne State University Press; 1995.

10. Hayden D, Square P. Verbal motor production assessment for children. San Antonio: The Psychological Corporation; 1997.

11. Strand EA, McCauley RJ, Weigand SD, Stoeckel RE, Baas BS. A motor speech assessment for children with severe speech disorders: reliability and validity evidence. J Speech Lang Hear Res. 2013;56(2):505-20. http://dx.doi.org/10.1044/1092-4388(2012/12-0094). PMid:23275421.

12. Preston JL, Edwards ML. Phonological processing skills of adolescents with residual speech sound errors. Lang Speech Hear Serv Sch. 2007;38(4):297-308. http://dx.doi.org/10.1044/0161-1461(2007/032). PMid:17890510.

13. Shriberg LD, Fourakis M, Hall S, Karlsson H, Lohmeier HL, McSweeny JL, et al. Extensions to the Speech Disorders Classification System (SDCS). Clin Linguist Phon. 2010;24(10):795-824. http://dx.doi.org /10.3109/02699206.2010.503006. PMid:20831378.

14. Marquardt TP, Jacks A, Davis BL. Token-to-token variability in developmental apraxia of speech: three longitudinal case studies. Clin Linguist Phon. 2004;18(2):127-44. http://dx.doi.org/10.1080/026992 00310001615050 . PMid:15086134.

15. Preston JL, Koenig LL. Phonetic variability in residual speech sound disorders: exploration of subtypes. Top Lang Disord. 2011;31(2):168-84. http://dx.doi.org/10.1097/TLD.0b013e318217b875. PMid:23087533.

16. Thoonen G, Maassen B, Gabreëls F, Schreuder R. Validity of maximum performance tasks to diagnose motor speech disorders in children. Clin Linguist Phon. 1999;13(1):1-23. http://dx.doi. org/10.1080/026992099299211.

17. Keske-Soares M, Uberti LB, Gubiani MB, Gubiani MB, Ceron MI, Pagliarin KC. Performance of children with speech sound disorders in the dynamic evaluation of motor speech skills. CoDAS. 2018;30(2):e20170037. http://dx.doi.org/10.1590/2317-1782/20182017037. PMid:29791618.

18. Tükel Ş, Björelius H, Henningsson G, McAllister A, Eliasson AC. Motor functions and adaptive behaviour in children with childhood apraxia of speech. Int J Speech Lang Pathol. 2015;17(5):470-80. http:// dx.doi.org/10.3109/17549507.2015.1010578. PMid:25740430.
19. Gomez M, McCabe P, Jakielski K, Purcell A. Treating childhood apraxia of speech with the kaufman speech to language protocol: a phase i pilot study. Lang Speech Hear Serv Sch. 2018;49(3):524-36. http://dx.doi.org/10.1044/2018_LSHSS-17-0100. PMid:29625432.

20. Iuzzini J, Forrest K. Evaluation of a combined treatment approach for childhood apraxia of speech. Clin Linguist Phon. 2010;24(4-5):335-45. http://dx.doi.org/10.3109/02699200903581083. PMid:20345262.

21. Sealey LR, Giddens CL. Aerodynamic indices of velopharyngeal function in childhood apraxia of speech. Clin Linguist Phon. 2010;24(6):41730. http://dx.doi.org/10.3109/02699200903447947. PMid:20136498.

22. Aziz AA, Shohdi S, Osman DM, Habib EI. Childhood apraxia of speech and multiple phonological disorders in Cairo-Egyptian Arabic speaking children: Language, speech, and oro-motor differences. Int J Pediatr Otorhinolaryngol. 2010;74(6):578-85. http://dx.doi.org/10.1016/j. ijporl.2010.02.003. PMid:20202694.

23. Ballard KJ, Robin DA, McCabe P, McDonald J. A treatment for dysprosody in childhood apraxia of speech. J Speech Lang Hear Res. 2010;53(5):1227-45. http://dx.doi.org/10.1044/1092-4388(2010/090130). PMid:20798323.

24. Thomas DC, McCabe P, Ballard KJ. Combined clinician parent delivery of Rapid Syllable Transition (ReST) treatment for childhood apraxia of speech. Int J Speech Lang Pathol. 2018;20(7):683-98. http://dx.doi. org/10.1080/17549507.2017.1316423. PMid:28443686.

25. Preston JL, Leece MC, McNamara K, Maas E. Variable practice to enhance speech learning in ultrasound biofeedback treatment for childhood apraxia of speech: a single case experimental study. Am J Speech Lang Pathol. 2017;26(3):840-52. http://dx.doi.org/10.1044/2017 AJSLP-16-0155. PMid:28715554.

26. Dunn L, Dunn L. Peabody Picture vocabulary test-III. Circle Pines, MN: AGS; 1997.

27. Thomas DC, McCabe P, Ballard KJ, Lincoln M. Telehealth delivery of Rapid Syllable Transitions (ReST) treatment for childhood apraxia of speech. Int J Lang Commun Disord. 2016;51(6):654-71. http://dx.doi. org/10.1111/1460-6984.12238. PMid:27161038.

28. Murray E, McCabe P, Ballard KJ. A randomized controlled trial for children with childhood apraxia of speech comparing rapid syllable transition treatment and the nuffield dyspraxia Programme-third edition. J Speech Lang Hear Res. 2015;58(3):669-86. http://dx.doi. org/10.1044/2015 JSLHR-S-13-0179. PMid:25807891.

29. Kotby MN, Bassiouny S, El Zomor EM. Standardization of an articulation test. In: Proceedings of the 9th Annual Ain Shams Medical Congress; 1986; Egypt. Egypt: Ain Shams University; 1986.

30. Namasivayam AK, Pukonen M, Goshulak D, Hard J, Rudzicz F, Rietveld $\mathrm{T}$, et al. Treatment intensity and childhood apraxia of speech. Int J Lang Commun Disord. 2015;50(4):529-46. http://dx.doi.org/10.1111/14606984.12154. PMid:25581372.

31. Wilcox K, Morris S. Children's Speech Intelligibility Measure (CSIM). San Antonio: Psychological Corporation; 1999.

32. Osberger MJ, Robbins AM, Todd SL, Riley AI. Speech intelligibility of children with cochlear implants. Volta Review. 1994;96:169-80.

33. McCabe P, Macdonald-D'Silva AG, Van Rees LJ, Ballard KJ, Arciuli J. Orthographically sensitive treatment for dysprosody in children with childhood apraxia of speech using ReST intervention. Dev Neurorehabil. 2014;17(2):137-45. http://dx.doi.org/10.3109/175184 23.2014.906002. PMid:24694312.

34. Dale PS, Hayden DA. Treating speech subsystems in childhood apraxia of speech with tactual input: the PROMPT approach. Am J Speech Lang Pathol. 2013;22(4):644-61. http://dx.doi.org/10.1044/10580360(2013/12-0055). PMid:23813194. 
35. Hodge MM, Daniels J, Gotzke CL. TOCS+ intelligibility measures (Version 5.3) [Computer software]. Edmonton, Canada: University of Alberta; 2009.

36. Preston JL, Brick N, Landi N. Ultrasound biofeedback treatment for persisting childhood apraxia of speech. Am J Speech Lang Pathol. 2013;22(4):627-43. http://dx.doi.org/10.1044/1058-0360(2013/120139). PMid:23813207.

37. Maas E, Butalla CE, Farinella KA. Feedback frequency in treatment for childhood apraxia of speech. Am J Speech Lang Pathol. 2012;21(3):239-57. http://dx.doi.org/10.1044/1058-0360(2012/11-0119). PMid:22442284.

38. Murray E, McCabe P, Ballard KJ. A comparison of two treatments for childhood apraxia of speech: methods and treatment protocol for a parallel group randomised control trial. BMC Pediatr. 2012;12(1):112. http://dx.doi.org/10.1186/1471-2431-12-112. PMid:22863021.

39. Newmeyer AJ, Aylward C, Akers R, Ishikawa K, Grether S, deGrauw T, et al. Results of the Sensory Profile in children with suspected childhood apraxia of speech. Phys Occup Ther Pediatr. 2009;29(2):20318. http://dx.doi.org/10.1080/01942630902805202. PMid:19401932.

40. Velleman SL. Phonotactic therapy. Semin Speech Lang. 2002;23(1):4356. http://dx.doi.org/10.1055/s-2002-23510. PMid:11938490.

41. Goldman R, Fristoe M. Goldman fristoe test of articulation. 2nd ed. Circle Pines, MN: AGS; 2000.

42. Dodd B, Crosbie S, Zhu H, Holm A, Ozanne A. The diagnostic evaluation of articulation and phonology. London: Psych-Corp; 2002.

43. Gozzard H, Baker E, McCabe P. Children's productions of polysyllabic words. ACQuiring Knowledge in Speech. Language and Hearing. 2006;8(3):113-6.

44. Robbins J, Klee T. Clinical assessment of oropharyngeal motor development in young children. J Speech Hear Disord. 1987;52(3):2717. http://dx.doi.org/10.1044/jshd.5203.271. PMid:3455449.

45. McCauley RJ, Strand EA. A review of standardized tests of nonverbal oral and speech motor performance in children. Am J Speech Lang
Pathol. 2008 Feb;17(1):81-91. http://dx.doi.org/10.1044/10580360(2008/007). PMid:18230815.

46. Gathercole SE, Baddeley AD. The children's test of nonword repetition. London: Psychological Corporation; 1996.

47. Shriberg LD, Lohmeier HL, Campbell TF, Dollaghan CA, Green JR, Moore CA. A nonword repetition task for speakers with misarticulations: the Syllable Repetition Task (SRT). J Speech Lang Hear Res. 2009;52(5):1189-212. http://dx.doi.org/10.1044/1092-4388(2009/080047). PMid:19635944.

48. Rvachew S, Hodge M, Ohberg A. Obtaining and interpreting maximum performance tasks from children: A tutorial. J Speech Lang Pathol Audiol. 2005;29(4):146-57.

49. Peppé S, McCann J. Assessing intonation and prosody in children with atypical language development: the PEPS-C test and the revised version. Clin Linguist Phon. 2003;17(4-5):345-54. http://dx.doi.org/ 10.1080/0269920031000079994. PMid:12945610.

50. Gubiani MB, McCauley RJ, Pagliarin KC, Keske-Soares M. Adaptation of the dynamic evaluation of motor speech skill from English to Brazilian Portuguese. Folia Phoniatr Logop. 2018. No prelo.

51. Shriberg LD, Potter NL, Strand EA. Childhood apraxia of speech in children and adolescents with galactosemia. In: American SpeechLanguage-Hearing Association National Convention; 2009; New Orleans, LA. Rockville: ASHA; 2009.

52. Coêlho JF. Apraxia de fala $\times$ desvio fonológico: aspectos linguísticos e análise acústica da fala na síndrome de down [tese]. João Pessoa: Curso de Linguística, Universidade Federal da Paraíba; 2018.

53. Navarro PR, Silva PMVA, Bordin SMS. Apraxia de fala na infầncia: para além das questões fonéticas e fonológicas. Distúrb Comun. 2018;30(3):475524. http://dx.doi.org/10.23925/2176-2724.2018v30i3p-475-489.

54. Wiig E, Secord WA, Semel E. The clinical evaluation of language fundamentals: preschool. 2nd ed. San Antonio: Harcourt Assessment; 2004. 